\title{
Vaccination control of an epidemic model with time delay and its application to COVID-19
}

\author{
Shidong Zhai • Guoqiang Luo • Tao Huang • \\ Xin Wang • Junli Tao $D$ Ping Zhou
}

Received: 7 January 2021 / Accepted: 8 May 2021 / Published online: 28 May 2021

(C) The Author(s), under exclusive licence to Springer Nature B.V. 2021

\begin{abstract}
This paper studies an SEIR-type epidemic model with time delay and vaccination control. The vaccination control is applied when the basic reproduction number $R_{0}>1$. The vaccination strategy is expressed as a state delayed feedback which is related to the current and previous state of the epidemic model, and makes the model become a linear system in new coordinates. For the presence and absence of vaccination control, we investigate the nonnegativity and boundedness of the model, respectively. We obtain some sufficient conditions for the eigenvalues of the linear system such that the nonnegativity of the epidemic model can be guaranteed when the vaccination strategy is applied. In addition, we study the stability of disease-free equilibrium when $R_{0}<1$ and the persistent of disease when $R_{0}>1$. Finally, we use the obtained theoretical results to simulate the vaccination strategy to control the spread of COVID-19.
\end{abstract}

Keywords Epidemic model - Time delay · State delayed feedback $\cdot$ Vaccination

S. Zhai · G. Luo $\cdot$ T. Huang $\cdot$ X. Wang

School of Automation, Chongqing University of Posts and

Telecommunications, Chongqing 400065, China

J. Tao $(\bowtie)$

Chongqing University Cancer Hospital,

Chongqing 400030, China

e-mail: taojunli0712@163.com

P. Zhou

School of Science, Chongqing University of Posts and Telecommunications, Chongqing 400065, China

\section{Introduction}

The outbreak of COVID-19 has caused a large number of deaths and economic losses to the world [1-4]. At present, many countries have not effectively controlled the epidemic, and the worst is that there are many asymptomatic infections [5-8]. Asymptomatic infections can transmit the virus to others, but they do not have any symptoms. In fact, the presence of asymptomatic infections is not unique to COVID-19, and many diseases have this property, such as EpsteinBarr virus, HIV, typhoid fever, etc. However, unlike other diseases having asymptomatic infections, the high infectiousness and a large number of asymptomatic infections make it difficult to control the spread of COVID-19 [8]. In order to effectively control the epidemic, various effective vaccines are the best choice. For COVID-19, many vaccines have entered phase III clinical trials [9-11]. Appropriate mathematical models and vaccination strategies are currently needed.

This paper introduces an SEIR type epidemic model with time delay, and a vaccination control is provided to make the removed-by-immunity population asymptotically track the whole population. The model assumes that there are many asymptomatic infections and exists an incubation period during which the strain of disease develops in the asymptomatic infections and it is only after that time that the asymptomatic infections become contagious. These two assumptions are suitable for COVID-19. Time delay is a common phenomenon in 
the spread of various diseases [12-15]. Since Cooke studied an SIR model with time delay which is caused by a vector [12], many epidemic models began to consider the factor of time delay in order to better simulate the spread of different diseases, such as SIS [16-18], SEIR [19-21] and SEIRS models [22-24]. The above epidemic models cannot describe the case that there are many asymptomatic infections. The two main factors causing time delay are (1) the latency of infected vector becoming infectious [12] and (2) the latency of the infected host becoming infectious. In addition, the time delay may occur in other scenarios, such as vaccination period and immune period [24].

Vaccination is the most effective way to completely control the spread of various diseases, such as smallpox [25] and measles [26]. When there exist various effective vaccines, appropriate vaccination strategy is needed to improve the efficacy of vaccination [27-29]. Since the epidemic models are nonlinear, many nonlinear control methods are used to design appropriate vaccination strategy [30-36]. For example, in [30], the authors considered an SEIR epidemic model and proposed a vaccination strategy based on feedback linearization techniques. A continuous linear vaccination control was proposed for SEIR model [31]. In [32], the impulsive vaccination was designed for cholera epidemics. The impulsive vaccination strategy was also studied for other cases, such as SIR epidemic model [33] and SEIR epidemic model with time delay [34]. The sliding mode control has been used to design vaccination strategy for SEIR epidemic model [36]. Although there are many vaccination strategies for various epidemic models, they cannot be directly used for proposed epidemic model with time delay. This paper will use feedback linearization technique to design a switching vaccination strategy for the proposed model.

The main contributions of this paper include the following: (1) We propose an SEIR type epidemic model with time delay based on the properties of COVID19 (high infectiousness and a large number of asymptomatic infections). When there do not exist vaccination control, we analyze the stability of the disease-free equilibrium and persistence of the disease. (2) Based on feedback linearization technique, we design a switching vaccination strategy for the proposed model, and obtain sufficient conditions for the nonnegativity of the model when the vaccination strategy is applied. (3) We simulate the vaccination strategy to control the
Table 1 Parameters of the epidemic model (1)

\begin{tabular}{ll}
\hline Parameter & Description \\
\hline$N_{0}$ & $\begin{array}{c}\text { The total population at the initial } \\
\text { time } \\
\text { Natural mortality rate } \\
\mu\end{array}$ \\
$\mu^{I}$ & $\begin{array}{l}\text { The death rate of populations } \\
\text { occurring in } I \text { compartment }\end{array}$ \\
$\alpha$ & The infection coefficient \\
$\beta$ & The rate of exposed class who \\
& become infective \\
$\gamma$ & The recovery rate of infective \\
& population
\end{tabular}

spread of COVID-19 and show the effectiveness of the obtained results.

The paper is organized as follows: Section 2 presents the problem statement and some basic properties of the model. Section 3 shows the stability of diseasefree equilibrium when $R_{0}<1$, and the persistence of disease when $R_{0}>1$. Section 4 provides a vaccination strategy for the proposed model. Section 5 simulates the vaccination control of COVID-19. Section 6 collects our conclusions.

\section{Problem statement and basic properties}

Motivated by Refs. [15,31], suppose that the total population $N(t)$ can be divided into four compartments, that is, $S(t), E(t), I(t)$, and $R(t)$, where $S(t), E(t)$, $I(t), R(t)$ denote the susceptible, exposed, infectious, recovered individuals at time $t$, respectively. Consider the SEIR-type epidemic model with time delay and vaccination control which is presented as following functional differential equation:

$$
\left\{\begin{array}{l}
\dot{S}=\mu N_{0}(1-u(t))-\frac{\alpha S(t) I(t-\tau)}{N_{0}}-\mu S+\delta R, \\
\dot{E}=\frac{\alpha S(t) I(t-\tau)}{N_{0}}-(\beta+\mu) E, \\
\dot{I}=\beta E-\left(\gamma+\mu^{I}\right) I, \\
\dot{R}=\mu N_{0} u(t)+\gamma I-(\delta+\mu) R,
\end{array}\right.
$$

where all parameters are nonnegative (see Table 1 for details), $u(t)$ is a vaccination control needed to design. $u(t)$ can be seen as a vaccination rate $[13,28]$. The flow diagram for model (1) is presented in Fig. 1. This epidemic model involves the following assumptions: 


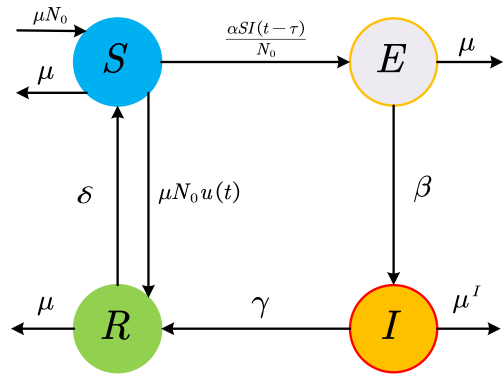

Fig. 1 The flow diagram for model (1)

(1) There exists a vector between susceptible and exposed individuals. The vector will become infectious after a time $\tau>0$. The susceptible population in $S$ enter into $E$ when they come into contact with infected vectors.

(2) The population in $E$ move to $I$ after the average latent time $\beta^{-1}$.

(3) Part of the recovered people become susceptible again to the disease.

(4) The death rate in $I$ is large than the natural death rate.

Note that the vector may be an animal (mosquito) [12] or a human (asymptomatic infection) [5]. Based on Assumption 1), in model (1), the total number of infections per unit of time at time $t$ is given by $\frac{\alpha S(t) I(t-\tau)}{N_{0}}$, which is called "true-mass action model" [31]. The model (1) can be used to describe the case that there exist many asymptomatic infections, such as COVID19. In Sect. 5, we will use our model to simulate the second outbreak of COVID-19 in Italy and show the effectiveness of the proposed vaccination control.

Let $\mathbb{R}^{n}$ denote the set of all real $n$-dimensional column vectors, and $\mathbb{R}_{+}^{n}:=\left\{x \in \mathbb{R}^{n}: x_{i} \geq\right.$ $0, i=1, \ldots, n\}$. Let $\mathscr{C}:=\mathscr{C}\left([-\tau, 0], \mathbb{R}^{4}\right)$ be the Banach space of continuous functions mapping the interval $[-\tau, 0]$ into $\mathbb{R}^{4}$ with norm $\|\phi\|=$ $\sum_{i=1}^{4}\left(\max _{\theta \in[-\tau, 0]}\left|\phi_{i}(\theta)\right|\right)$, where $\phi=\left[\phi_{1}, \ldots, \phi_{4}\right] \in$ $\mathscr{C}$. Let $\mathscr{C}^{+}:=\mathscr{C}\left([-\tau, 0], \mathbb{R}_{+}^{4}\right)$ which is a cone of $\mathscr{C}$. We consider the solutions of system (1) with initial conditions

$$
\begin{aligned}
& S_{0}=\phi_{1}(s) \geq 0, \quad E_{0}=\phi_{2}(s) \geq 0, \\
& I_{0}=\phi_{3}(s) \geq 0, \quad R_{0}=\phi_{4}(s) \geq 0,
\end{aligned}
$$

where $\phi(s) \in \mathscr{C}^{+}$.
One can know from (1) that

$$
\begin{aligned}
\dot{N} & =\mu N_{0}-\mu S-\mu E-\mu^{I} I-\mu R, \\
& \leq \mu N_{0}-\min \left\{\mu, \mu^{I}\right\} N .
\end{aligned}
$$

If the death rate $\mu=\mu^{I}$, then $\dot{N}=\mu\left(N_{0}-N\right)$ and the total population $N(t)=N(0)=N_{0}, \forall t \geq 0$. Generally speaking, $\mu^{I}$ is slightly larger than the death rate $\mu$. Hence, $N \leq \frac{\mu-e^{-\psi t}(\mu-\psi)}{\psi} N_{0}$, where $\psi=$ $\min \left\{\mu, \mu^{I}\right\}$. Since $\mu-\psi \approx 0$, the total population $N$ can be regarded as constant in a short time.

In this paper, we want to design a vaccination strategy to make the infective population approach zero, and the removed-by-immunity population asymptotically track the whole population.

When $u(t) \equiv 0$, that is, there does not exist vaccination control, the following theorem shows that the system (1) has positively invariant set

$$
\begin{aligned}
\Gamma= & \left\{[S(t), E(t), I(t), R(t)]^{\top} \in \mathbb{R}_{+}^{4}: S(t)\right. \\
& \left.+E(t)+I(t)+R(t) \leq N_{0}\right\} .
\end{aligned}
$$

Theorem 1 Consider the system (1) with initial condition (2). Then, the system (1) has positively invariant set $\Gamma$.

Proof Let $g(\phi)=\left[g_{1}(\phi), g_{2}(\phi), g_{3}(\phi), g_{4}(\phi)\right]^{\top}$, where $\forall \phi \in \mathscr{C}^{+}$, and

$g_{1}(\phi)=\mu N_{0}-\frac{\alpha \phi_{1}(0) \phi_{3}(-\tau)}{N_{0}}-\mu \phi_{1}(0)+\delta \phi_{4}(0)$,

$g_{2}(\phi)=\frac{\alpha \phi_{1}(0) \phi_{3}(-\tau)}{N_{0}}-(\beta+\mu) \phi_{2}(0)$,

$g_{3}(\phi)=\beta \phi_{2}(0)-\left(\gamma+\mu^{I}\right) \phi_{3}(0)$,

$g_{4}(\phi)=\gamma \phi_{3}(0)-(\delta+\mu) \phi_{4}(0)$.

Then, for each compact set $\Omega \in \mathbb{R} \times \mathscr{C}^{+}, g(\phi)$ is continuous and Lipschitz in $\Omega$. Hence, there exists a unique solution of system (1) through $(0, \phi)[37$, Theorem 2.2.3]. Since $\phi=[0,0,0,0]^{\top}$ is a solution of system (1), all solutions of system (1) are nonnegative at maximal interval. One can see from the above discussion that the total population $N(t) \leq N_{0}, \forall t \geq 0$. Hence, all solutions of system (1) are bounded, and exist on the interval $[0, \infty)$. Thus, the set $\Gamma$ is positively invariant for system (1). 


\section{Stability analysis}

This section will introduce the basic reproduction number of system (1), present the stability analysis of the disease-free equilibrium, and show that the disease is persistent when the basic reproduction number is greater than one.

When $u(t) \equiv 0$, the disease-free equilibrium of model (1) is $P_{0}=\left[N_{0}, 0,0,0\right]^{\top}$. Based on Ref. [14], let $\mathscr{F}$ and $\mathscr{Y}$ denote the rate of secondary infections increase and the rate of disease progression, death, recovery decrease, respectively. Then,

$\mathscr{F}=\left[\begin{array}{c}\frac{\alpha S(t) I(t-\tau)}{N_{0}} \\ 0\end{array}\right], \quad \mathscr{Y}=\left[\begin{array}{c}(\beta+\mu) E \\ -\beta E+\left(\gamma+\mu^{I}\right) I\end{array}\right]$.

The next generation matrix of system (1) can be presented as $F Y^{-1}$, where $F=\left(\frac{\partial \mathscr{F}}{\partial x}\left(P_{0}\right)\right), Y=$ $\left(\frac{\partial \mathscr{Y}}{\partial x}\left(P_{0}\right)\right), x=[E, I]^{\top}$.

Hence,

$F=\left[\begin{array}{cc}0 & \frac{\alpha S_{0}}{N_{0}} \\ 0 & 0\end{array}\right], \quad Y=\left[\begin{array}{cc}\beta+\mu & 0 \\ -\beta & \gamma+\mu^{I}\end{array}\right]$,

and the next-generation matrix is $K=F Y^{-1}$,

$K=F V^{-1}=\left[\begin{array}{cc}\frac{\alpha S_{0} \beta}{N_{0}(\beta+\mu)\left(\gamma+\mu^{I}\right)} & \frac{\alpha S_{0}}{\gamma+\mu^{I}} \\ 0 & 0\end{array}\right]$.

Then, the basic reproduction number equals the spectral radius of matrix $K$, that is,

$R_{0}=\rho(K)=\frac{\alpha \beta}{(\beta+\mu)\left(\gamma+\mu^{I}\right)}$.

Theorem 2 Consider the system (1). If $R_{0}<1$, then the disease-free equilibrium $P_{0}$ is globally asymptotically stable. If $R_{0}>1$, then the disease-free equilibrium $P_{0}$ is unstable.

Proof We will use the method of fluctuations [38, Lemma 4.2] to show that

$\lim _{t \rightarrow \infty} \sup S(t)=N_{0}, \lim _{t \rightarrow \infty} \sup E(t)=0$,

$\lim _{t \rightarrow \infty} \sup I(t)=0, \lim _{t \rightarrow \infty} \sup S(t)=0$.

For simplicity, let $\bar{S}=\lim _{t \rightarrow \infty} \sup S(t), \bar{E}=$ $\lim _{t \rightarrow \infty} \sup E(t), \quad \bar{I}=\lim _{t \rightarrow \infty} \sup I(t)$, $\bar{R}=\lim _{t \rightarrow \infty} \sup R(t)$. By Lemma 4.2 [38], there exist four sequences $\pi_{n}^{i} \rightarrow \infty, n \rightarrow \infty, i=1,2,3,4$ such that

$$
\lim _{n \rightarrow \infty} S\left(\pi_{n}^{1}\right)=\bar{S}, \dot{S}\left(\pi_{n}^{1}\right)=0,
$$

$\lim _{n \rightarrow \infty} E\left(\pi_{n}^{2}\right)=\bar{E}, \dot{E}\left(\pi_{n}^{2}\right)=0$

$\lim _{n \rightarrow \infty} I\left(\pi_{n}^{3}\right)=\bar{I}, \dot{I}\left(\pi_{n}^{3}\right)=0$,

$\lim _{n \rightarrow \infty} R\left(\pi_{n}^{4}\right)=\bar{R}, \dot{R}\left(\pi_{n}^{4}\right)=0$.

Then, one has the following inequalities from (1)

$$
\left\{\begin{array}{l}
0 \leq \mu N_{0}-\mu \bar{S}+\delta \bar{R}, \\
0 \leq \frac{\alpha \bar{S} \bar{I}}{N_{0}}-(\beta+\mu) \bar{E}, \\
0 \leq \beta \bar{E}-\left(\gamma+\mu^{I}\right) \bar{I}, \\
0 \leq \gamma \bar{I}-(\delta+\mu) \bar{R} .
\end{array}\right.
$$

The third inequality of (4) implies $\bar{E} \leq \frac{\left(\gamma+\mu^{I}\right) \bar{I}}{\beta}$. Since $\bar{S} \leq N_{0}$, the second inequality of (4) implies $0 \leq \alpha \bar{I}-$ $(\beta+\mu) \bar{E}$. Hence, $0 \leq\left(R_{0}-1\right) \bar{I}$. Since $R_{0}<1$, $\bar{I}=\bar{E}=0$. The fourth inequality of (4) implies $\bar{R}=0$. The first inequality of (4) implies $\bar{S}=N_{0}$. Hence, the disease-free equilibrium $P_{0}$ is globally attractive.

One can know from Theorem 2.1 in [14] that the disease-free equilibrium $P_{0}$ is locally stable when $R_{0}<$ 1 , and the disease-free equilibrium $P_{0}$ is unstable when $R_{0}>1$. Hence, if $R_{0}<1$, then the disease-free equilibrium $P_{0}$ is globally asymptotically stable.

When $R_{0}>1$, the disease will persist in the population. The following theorem will provide the detail proof.

Theorem 3 Consider the system (1). If $R_{0}>1$, then the disease is uniformly persistent, that is, there exists a positive number $b$ such that $\lim _{t \rightarrow \infty}$ inf $I(t) \geq b$.

Proof For the system(1), let $x(t, \phi)=[S(t), E(t), I(t)$, $R(t)]^{\top}$ be the solution of system (1) with initial condition $\phi \in \mathscr{C}^{+}$. Because $S(t) \rightarrow N_{0}$ as $x(t, \phi) \rightarrow P_{0}$, for sufficiently small $v>0$ there exists a neighborhood of $P_{0}$ such that $N_{0}-v<S(t)<N_{0}+v$.

First, we will show that there exists $\varepsilon(v)>0$ such that $\lim _{t \rightarrow \infty} \sup \left\|x(t, \phi)-P_{0}\right\| \geq \varepsilon(v)$ for any $\phi \in$ $\mathscr{C}^{+}$. We will prove it by contradiction. If the above condition does not hold, then there exists $\phi \in \mathscr{C}^{+}$such that $\left\|x(t, \phi)-P_{0}\right\|<\varepsilon(v)$. Hence, there exists $T>0$ such that $\left|S(t)-N_{0}\right|<\varepsilon(v),|E(t)|<\varepsilon(v),|I(t)|<$ $\varepsilon(v)$, and $|R(t)|<\varepsilon(v)$ for $t>T$. One can obtain from the second and third equation of (1) that

$$
\begin{aligned}
(\beta+\mu) \dot{I}+\beta \dot{E}= & \frac{\alpha \beta S(t) I(t-\tau)}{N_{0}} \\
& -(\beta+\mu)\left(\gamma+\mu^{I}\right) I .
\end{aligned}
$$


By Lemma 4.2 [38], there exist a sequence $\pi_{n} \rightarrow$ $\infty, n \rightarrow \infty$ such that $\lim _{n \rightarrow \infty} E\left(\pi_{n}\right)=0, \dot{E}\left(\pi_{n}\right)=$ 0 . Hence, the following inequality holds

$$
\begin{aligned}
(\beta+\mu) \dot{I}\left(\pi_{n}\right)> & \frac{\alpha \beta\left(N_{0}-\nu\right) I\left(\pi_{n}-\tau\right)}{N_{0}} \\
& -(\beta+\mu)\left(\gamma+\mu^{I}\right) I\left(\pi_{n}\right) .
\end{aligned}
$$

Consider the following equation

$$
\begin{aligned}
(\beta+\mu) \dot{I}(t)= & \frac{\alpha \beta\left(N_{0}-v\right) I(t-\tau)}{N_{0}} \\
& -(\beta+\mu)\left(\gamma+\mu^{I}\right) I(t) .
\end{aligned}
$$

The characteristic equation of (7) is

$$
\begin{aligned}
\Theta(\lambda):= & (\beta+\mu) \lambda-\frac{\alpha \beta\left(N_{0}-v\right) e^{-\lambda \tau}}{N_{0}} \\
& +(\beta+\mu)\left(\gamma+\mu^{I}\right)=0 .
\end{aligned}
$$

When $v=0$ and $R_{0}>1, \Theta(0)=(\beta+\mu)\left(\gamma+\mu^{I}\right)(1-$ $\left.R_{0}\right)<0$. It is easy to see that $\Theta(\lambda)$ is a continuous function for $\lambda \geq 0$ and increases to $\infty$ as $\lambda \rightarrow \infty$. Hence, for Eq. (8) there exists a positive root. Suppose that $\bar{\lambda}(v)$ is the principle eigenvalue. Thus, $\bar{\lambda}(0)>0$. Since $\bar{\lambda}(v)$ is a continuous function for $v$, one can get $\bar{\lambda}(v)>0$ for sufficiently small $v>0$. Hence, $c e^{\bar{\lambda}(v) t}$ $(c>0)$ is a solution of Eq. (7). By the comparison theorem, one can obtain that there exists $a>0$ such that $I\left(\pi_{n}\right) \geq a c e^{\bar{\lambda}(v) \pi_{n}}$. Hence, $I\left(\pi_{n}\right) \rightarrow \infty$ as $n \rightarrow \infty$, which is a contraction for the fact that $I(t)$ is bounded.

Let $\omega(\phi)$ be the omega limit set of $x(t, \phi)$. Define $M=\left\{\phi \in \mathscr{C}^{+}: x(t, \phi) \in \partial \mathscr{C}^{+}, t \geq 0\right\}$. One can obtain from the third equation of (1) that

$\dot{I}(t) \geq-\left(\gamma+\mu^{I}\right) I(t)$.

Hence, if there exists $T \geq 0$ such that $I(T)>0$, then $I(t)>0$ for all $t \geq T$. Thus, $I(t)=0$ when $\phi \in M$. For any $\phi \in M \omega(\phi)=P_{0}$, and there does not exist a cycle in $M$. With the similar proof of Theorem 3.1 [14], there exists $b>0$ such that $\lim _{t \rightarrow \infty}$ inf $I(t) \geq b$.

\section{Vaccination control strategy}

This section will design a vaccination control $u(t)$ which makes the removed-by-immunity population asymptotically track the whole population. $u(t)$ will present as a state delayed feedback.
Since $N=S+E+I+R$, we can obtain that $\dot{N}=\mu N_{0}-\mu N+\left(\mu-\mu^{I}\right) I$.

Hence, $N=e^{-\mu t} N_{0}+\int_{0}^{t} e^{-\mu(t-s)}\left[\mu N_{0}+(\mu-\right.$ $\left.\left.\mu^{I}\right) I\right] d s$. Because $R=N-S-E-I$ and $N$ can be presented as a function of $I$, we just need to consider the first three equations about $S, E, I$ in model (1).

The system (1) can be rewritten as the following nonlinear delay system:

$\left\{\begin{array}{l}\dot{x}=f(x(t), x(t-\tau))+g(x(t)) u(t), \\ y(t)=h(x(t)),\end{array}\right.$

where $x(t)=[S(t), E(t), I(t)]^{\top} \in \mathbb{R}_{+}^{3}$,

$$
\begin{aligned}
& f(x(t), x(t-\tau)) \\
& =\left[\begin{array}{c}
\mu N_{0}-\frac{\alpha S(t) I(t-\tau)}{N_{0}}-\mu S+\delta(N-S-E-I) \\
\frac{\alpha S(t) I(t-\tau)}{N_{0}}-(\beta+\mu) E \\
\beta E-\left(\gamma+\mu^{I}\right) I
\end{array}\right], \\
& g(x(t))=\left[\begin{array}{c}
-\mu N_{0} \\
0 \\
0
\end{array}\right], \quad h(x(t))=I(t) .
\end{aligned}
$$

In the sequel, let $x_{i \tau}(t)=x(t-i \tau), u_{i \tau}(t)=u(t-i \tau)$, $i=0,1, \ldots x_{i \tau}$ is defined for $t \geq(i-1) \tau$, and $u_{i \tau}$ is defined for $t \geq i \tau$.

Definition 1 Assume that for system (9), there exists an integer $r$ such that, for every $X$ in $\Omega_{r} \subseteq \mathbb{R}^{3(r+1)}$, the following conditions are satisfied

$L_{G} L_{F}^{k} H(X)=0, \quad k=0,1, \ldots, r-2$,

$L_{G} L_{F}^{r-1} H(X)\left[\begin{array}{c}1 \\ 0 \\ \vdots \\ 0\end{array}\right] \neq 0$,

where

$$
\begin{gathered}
X=\left[\begin{array}{c}
x \\
x_{\tau} \\
\vdots \\
x_{r \tau}
\end{array}\right], \quad \bar{X}=\left[\begin{array}{c}
x \\
x_{\tau} \\
\vdots \\
x_{(r-1) \tau}
\end{array}\right], \\
F(X)=\left[\begin{array}{c}
f\left(x, x_{\tau}\right) \\
f\left(x_{\tau}, x_{2 \tau}\right) \\
\vdots \\
f\left(x_{(r-1) \tau}, x_{r \tau}\right)
\end{array}\right],
\end{gathered}
$$


$G(X)=\operatorname{diag}\left\{g(x), \ldots, g\left(x_{(r-1) \tau}\right)\right\}$,

$H(X)=h(x)$,

$L_{F}^{0} H(X)=H(X)$,

$L_{F}^{k} H(X)=\left(\frac{\mathrm{d}}{\mathrm{d} \bar{X}} L_{F}^{k-1} H\right) F(X)$,

$L_{G} L_{F}^{k} H(X)=\left(\frac{\mathrm{d}}{\mathrm{d} \bar{X}} L_{F}^{k} H\right) G(X)$,

then the delay relative degree of system (9) is equal to $r$ in $\Omega_{r}$.

By a simple calculation, $L_{G} L_{F}^{k} H(X)=0, k=0,1$ and $L_{G} L_{F}^{2} H(X)[1,0,0]^{\top}=-\mu \beta \alpha I_{\tau}$. Hence, the delay relative degree of system (9) is 3 in $\Omega_{3}:=\{X$ : $\left.x=[S, E, I]^{\top}, I_{\tau} \neq 0\right\}$. Then, we can obtain that

$y^{(k)}(t)=L_{F}^{k} H(X), \quad k=0,1,2$,

$y^{(3)}(t)=L_{F}^{3} H(X)+\Gamma(X) u$,

where

$\Gamma(X)=L_{G} L_{F}^{2} H(X)\left[\begin{array}{l}1 \\ 0 \\ 0\end{array}\right]$.

Let $z(t)=[y(t), \dot{y}(t), \ddot{y}(t)]^{\top}$. Then, we have

$\dot{z}(t)=\left[\begin{array}{lll}0 & 1 & 0 \\ 0 & 0 & 1 \\ 0 & 0 & 0\end{array}\right] z(t)+\left[\begin{array}{c}0 \\ 0 \\ L_{F}^{3} H(X)+\Gamma(X) u(t)\end{array}\right]$.

If we choose

$u(t)=\frac{-L_{F}^{3} H(X)+\left(k_{1} z_{1}(t)+k_{2} z_{2}(t)+k_{2} z_{3}(t)\right)}{\Gamma(X)}$,

then the equation (12) becomes

$\dot{z}(t)=\left[\begin{array}{ccc}0 & 1 & 0 \\ 0 & 0 & 1 \\ k_{1} & k_{2} & k_{3}\end{array}\right] z(t)$,

where the parameters $k_{1}, k_{2}$ and $k_{3}$ are needed to design.

The above analysis process can be presented in the following proposition:

Proposition 1 Consider the system (1) with time delay. If the state delayed feedback control law $u(t)$ defined as in (13), then one can obtain the closed-loop system (14).
Remark 1 Note that the control law $u(t)$ is well defined all $x \in \Omega_{3}:=\left\{X: x=[S, E, I]^{\top}, I_{\tau} \neq 0\right\}$. However, the disease may be considered eradicated from the population once the infectious population is smaller than one individual. Hence, the vaccination strategy can be switched off when $0<I(t) \leq \varepsilon<1$. In this sense, the efficient control law is given by

$u_{e}(t)= \begin{cases}u(t) & \text { if } 0 \leq t \leq t_{f} \\ 0 & \text { if } t>t_{f}\end{cases}$

where $t_{f}$ is defined as follows:

$t_{f} \triangleq \min \left\{t \in \mathbb{R}_{+}: I(t)<\varepsilon\right.$ for some $\left.0<\varepsilon<1\right\}$.

Remark 2 Note that although the vector $X$ is defined for $t \geq 2 \tau$, the control $u(t)$ is a function of $x$ and $x(t-\tau)$. Hence, the control $u_{e}(t)$ can be applied at $\tau$.

Remark 3 For linear system (14), its characteristic polynomial $P(s)=s^{3}-k_{3} s^{2}-k_{2} s-k_{1}=0$. Making $P(s)=\left(s+r_{1}\right)\left(s+r_{2}\right)\left(s+r_{3}\right)$. In order to make the linear system (14) asymptotically stable, the parameters $k_{1}, k_{2}, k_{3}$ should be chosen to make $r_{i}>0, i=1,2,3$. It can be get that $k_{1}=-r_{1} r_{2} r_{3}<0, k_{2}=-\left(r_{1} r_{2}+\right.$ $\left.r_{1} r_{3}+r_{2} r_{3}\right)<0, k_{3}=-\left(r_{1}+r_{2}+r_{3}\right)<0$.

When the control $u_{e}(t)$ is applied at $\tau$, we must guarantee that $S, E, I, R$ of system (1) are nonnegative. Hence, the parameters $k_{1}, k_{2}, k_{3}$ should be chosen to make $r_{i}>0, i=1,2,3$ satisfy the following theorem.

Theorem 4 Consider the SEIR type epidemic model (1) with an initial condition (2), and $I(0) \leq I(\tau)$. Suppose that the positive real numbers $r_{i}, i=1,2,3$ satisfy the following conditions:

(1) $r_{1}<\min \left\{\mu+\beta, \mu^{I}+\gamma\right\}, r_{2}=\mu^{I}+\gamma$, and $r_{3}>\max \left\{\mu+\beta, \mu^{I}+\gamma\right\}$.

(2) $r_{1}$ and $r_{3}$ satisfy the inequalities:

$$
\left\{\begin{array}{l}
\left(r_{3}-r_{1}\right)\left(r_{3}-\mu^{I}-\gamma\right)>\beta \alpha \\
r_{1}+r_{3}-\alpha-\mu-\left(\beta+\mu+\mu^{I}+\gamma\right) \geq 0,(17) \\
(\beta+\mu)^{2}+r_{1} r_{3}-\left(r_{1}+r_{3}\right)(\beta+\mu) \geq 0 .
\end{array}\right.
$$

Then, the application of control law (15) guarantees the nonnegativity of the SEIR-type epidemic model (1), and $u(t) \geq 1, \forall t \in\left[\tau, t_{f}\right]$.

Proof Because $z(t)=\left[\begin{array}{lll}y(t), & \dot{y}(t), & \ddot{y}(t)\end{array}\right]^{\top}$ and $y(t)=I(t), I(t)=c_{1} e^{-r_{1}(t-\tau)}+c_{2} e^{-r_{2}(t-\tau)}+$ 
$c_{3} e^{-r_{3}(t-\tau)}, \forall t \geq \tau$. The following equations can be obtained

$$
\begin{aligned}
& c_{1}+ c_{2}+c_{3}=I(\tau), \\
&-\left(c_{1} r_{1}+c_{2} r_{2}+c_{3} r_{3}\right)=\beta E(\tau)-\left(\mu^{I}+\gamma\right) I(\tau), \\
& c_{1} r_{1}^{2}+c_{2} r_{2}^{2}+c_{3} r_{3}^{2} \\
&=\beta\left[\frac{\alpha S(\tau) I(0)}{N_{0}}-(\beta+\mu) E(\tau)\right] \\
&-\left(\gamma+\mu^{I}\right)\left[\beta E(\tau)-\left(\gamma+\mu^{I}\right) I(\tau)\right] .
\end{aligned}
$$

The above equations can be expressed in matrix form $R \cdot C=M$, where

$$
\begin{aligned}
& R=\left[\begin{array}{ccc}
1 & 1 & 1 \\
r_{1} & r_{2} & r_{3} \\
r_{1}^{2} & r_{2}^{2} & r_{3}^{2}
\end{array}\right], \quad C=\left[\begin{array}{c}
c_{1} \\
c_{2} \\
c_{3}
\end{array}\right], \\
& M=\left[\begin{array}{c}
I(\tau) \\
\left(\mu^{I}+\gamma\right) I(\tau)-\beta E(\tau) \\
\beta\left[\frac{\alpha S(\tau) I(0)}{N_{0}}-(\beta+\mu) E(\tau)\right]-\left(\gamma+\mu^{I}\right)\left(\beta E(\tau)-\left(\gamma+\mu^{I}\right) I(\tau)\right)
\end{array}\right] .
\end{aligned}
$$

The matrix $R$ is the Vandermonde matrix and its determinant is equal to $\left(r_{2}-r_{1}\right)\left(r_{3}-r_{1}\right)\left(r_{3}-r_{2}\right)$. Hence, $R$ is an invertible matrix when $r_{1} \neq r_{2} \neq r_{3}$. By direct calculation,

$$
\begin{aligned}
& R^{-1}=\left[\begin{array}{ccc}
\frac{r_{2} r_{3}}{\left(r_{3}-r_{1}\right)\left(r_{2}-r_{1}\right)} & \frac{-\left(r_{2}+r_{3}\right)}{\left(r_{3}-r_{1}\right)\left(r_{2}-r_{1}\right)} & \frac{1}{\left(r_{3}-r_{1}\right)\left(r_{2}-r_{1}\right)} \\
\frac{-r_{1} r_{3}}{\left(r_{2}-r_{1}\right)\left(r_{3}-r_{2}\right)} & \frac{r_{1}+r_{3}}{\left(r_{2}-r_{1}\right)\left(r_{3}-r_{2}\right)} & \frac{-1}{\left(r_{2}-r_{1}\right)\left(r_{3}-r_{2}\right)} \\
\frac{r_{1} r_{2}}{\left(r_{3}-r_{1}\right)\left(r_{3}-r_{2}\right)} & \frac{-\left(r_{1}+r_{2}\right)}{\left(r_{3}-r_{1}\right)\left(r_{3}-r_{2}\right)} & \frac{1}{\left(r_{3}-r_{1}\right)\left(r_{3}-r_{2}\right)}
\end{array}\right], \\
& C=\left[\begin{array}{c}
\frac{\left(r_{2} r_{3}+\left(\mu^{I}+\gamma\right)^{2}-\left(r_{2}+r_{3}\right)\left(\mu^{I}+\gamma\right)\right) I(\tau)+\beta\left(r_{2}+r_{3}-\mu-\mu^{I}-\gamma-\beta\right) E(\tau)+\beta \frac{\alpha S(\tau) I(0)}{N_{0}}}{\left(r_{3}-r_{1}\right)\left(r_{2}-r_{1}\right)} \\
-\frac{\left(r_{1} r_{3}+\left(\mu^{I}+\gamma\right)^{2}-\left(r_{1}+r_{3}\right)\left(\mu^{I}+\gamma\right)\right) I(\tau)+\beta\left(r_{1}+r_{3}-\mu-\mu^{I}-\gamma-\beta\right) E(\tau)+\beta \frac{\alpha S(\tau) I(0)}{N_{0}}}{\left(r_{3}-r_{2}\right)\left(r_{2}-r_{1}\right)} \\
\frac{\left(r_{1} r_{2}+\left(\mu^{I}+\gamma\right)^{2}-\left(r_{1}+r_{2}\right)\left(\mu^{I}+\gamma\right)\right) I(\tau)+\beta\left(r_{1}+r_{2}-\mu-\mu^{I}-\gamma-\beta\right) E(\tau)+\beta \frac{\alpha S(\tau) I(0)}{N_{0}}}{\left(r_{3}-r_{1}\right)\left(r_{3}-r_{2}\right)}
\end{array}\right] .
\end{aligned}
$$

If the condition (1) holds, then $c_{1}=\frac{\beta\left(r_{3}-\mu-\beta\right) E(0)+\beta \frac{\alpha S(0) I(-\tau)}{N}}{\left(r_{3}-r_{1}\right)\left(\mu+\gamma-r_{1}\right)}$ and $r_{3}>r_{2}>r_{1}$. Hence, $c_{1}>0$. In the sequel, we show that $I(t) \geq 0$.

Since the sign of $c_{2}$ and $c_{3}$ is uncertain, there are four cases: (i) $c_{2} \geq 0$ and $c_{3} \geq 0$, (ii) $c_{2} \geq 0$ and $c_{3}<0$, (iii) $c_{2}<0$ and $c_{3} \geq 0$, and (iv) $c_{2}<0$ and $c_{3}<0$.

For the case (i) and (ii), since $c_{2} \geq 0$, one can obtain $I(t)=c_{1} e^{-r_{1}(t-\tau)}+c_{2} e^{-r_{2}(t-\tau)}$
For the case (iv), that is $c_{2}<0$ and $c_{3}<0$, we have

$$
\begin{aligned}
I(t)= & {\left[I(\tau)-c_{2}-c_{3}\right] e^{-r_{1}(t-\tau)}+c_{2} e^{-r_{2}(t-\tau)} } \\
& +c_{3} e^{-r_{3}(t-\tau)} \\
= & I(\tau) e^{-r_{1}(t-\tau)}+c_{2}\left(e^{-r_{2}(t-\tau)}-e^{-r_{1}(t-\tau)}\right) \\
& +c_{3}\left(e^{-r_{3}(t-\tau)}-e^{r_{1}(t-\tau)}\right)
\end{aligned}
$$$$
\geq 0, \quad \forall t \geq 0 \text {. }
$$ 
Hence, for the cases (i)-(iv), $I(t) \geq 0, \forall t \geq 0$.

We will show that $E(t) \geq 0$ and $S(t) \geq 0$. We can obtain from the third equation of (1) that

$$
\begin{aligned}
E(t) & =\frac{\dot{I}(t)+\left(\mu^{I}+\gamma\right) I(t)}{\beta} \\
& =\sum_{i=1}^{3} \frac{c_{i}\left(\mu^{I}+\gamma-r_{i}\right) e^{-r_{i}(t-\tau)}}{\beta} .
\end{aligned}
$$

Taking $r_{2}=\mu^{I}+\gamma$, then

$E(t)=\frac{c_{1}\left(\mu^{I}+\gamma-r_{1}\right) e^{-r_{1}(t-\tau)}+c_{3}\left(\mu^{I}+\gamma-r_{3}\right) e^{-r_{3}(t-\tau)}}{\beta}$.

Hence, $c_{3}\left(\mu^{I}+\gamma-r_{3}\right)=\beta E(\tau)-c_{1}\left(\mu^{I}+\gamma-r_{1}\right)$. Then, one can obtain from (20) that

$$
\begin{aligned}
E(t)= & \frac{1}{\beta}\left[c_{1}\left(\mu^{I}+\gamma-r_{1}\right)\left(e^{-r_{1}(t-\tau)}-e^{-r_{3}(t-\tau)}\right)\right. \\
& \left.+\beta E(\tau) e^{-r_{3}(t-\tau)}\right] \\
\geq & 0, \quad \forall t \geq 0 .
\end{aligned}
$$

Similarly, one can obtain from the second equation of (1) that

$$
S(t)=\frac{N_{0}[\dot{E}+(\beta+\mu) E]}{\alpha I(t-\tau)} .
$$

Combining (20) and (21), one has

$$
\begin{aligned}
S(t)= & \frac{N_{0} c_{1}\left(\mu^{I}+\gamma-r_{1}\right)\left(\beta+\mu-r_{1}\right) e^{-r_{1}(t-\tau)}}{\alpha \beta I(t-\tau)} \\
& +\frac{N_{0} c_{3}\left(\mu^{I}+\gamma-r_{3}\right)\left(\beta+\mu-r_{3}\right) e^{-r_{3}(t-\tau)}}{\alpha \beta I(t-\tau)} .
\end{aligned}
$$

From the above equation, it follows that

$$
\begin{aligned}
& N_{0} c_{3}\left(\mu^{I}+\gamma-r_{3}\right)\left(\beta+\mu-r_{3}\right) \\
& =\alpha \beta I(0) S(\tau) \\
& \quad-N_{0} c_{1}\left(\mu^{I}+\gamma-r_{1}\right)\left(\beta+\mu-r_{1}\right) .
\end{aligned}
$$

Hence,
The nonnegativity of $R(t)$ is proven by using continuity arguments. If $R(t)$ has a negative value, then there is a point $t_{0}$ such that $R\left(t_{0}\right)=0$ when $R(0) \geq 0$. Then, it follows from the fourth equation of (1) that

$$
\begin{aligned}
\dot{R}\left(t_{0}\right)= & \mu N_{0}\left(p+u\left(t_{0}\right)\right)+\gamma I\left(t_{0}\right) \\
\geq & \gamma I\left(t_{0}\right)+\beta S\left(t_{0}\right) E\left(t_{0}-\tau\right) \\
& -\left[k_{3}+\alpha+\mu+\left(\beta+\mu+\mu^{I}+\gamma\right)\right. \\
& \left.+\left(\mu^{I}+\gamma\right)\right] S\left(t_{0}\right) \\
& +\left[\left(\beta+\mu+k_{3}\right)\left(\beta+\mu+\mu^{I}+\gamma\right)\right. \\
& \left.+\left(\mu^{I}+\gamma\right)^{2}-k_{2}\right] \frac{N_{0} E\left(t_{0}\right)}{\alpha I\left(t_{0}-\tau\right)} \\
& +\left[k_{2}\left(\mu^{I}+\gamma\right)-k_{1}-\left(\mu^{I}+\gamma\right)^{3}\right. \\
& \left.-k_{3}\left(\mu^{I}+\gamma\right)^{2}\right] \frac{N_{0} I\left(t_{0}\right)}{\alpha \beta I\left(t_{0}-\tau\right)} .
\end{aligned}
$$

Because $k_{1}=-r_{1} r_{2} r_{3}<0, k_{2}=-\left(r_{1} r_{2}+r_{1} r_{3}+\right.$ $\left.r_{2} r_{3}\right)<0, k_{3}=-\left(r_{1}+r_{2}+r_{3}\right)<0$ and $r_{2}=\mu^{I}+\gamma$, then one has

$$
\begin{aligned}
\dot{R}\left(t_{0}\right) \geq & \gamma I\left(t_{0}\right)+\beta S\left(t_{0}\right) E\left(t_{0}-\tau\right) \\
+ & {\left[r_{1}+r_{3}-\alpha-\mu-\left(\beta+\mu+\mu^{I}+\gamma\right)\right] S\left(t_{0}\right) } \\
+ & {\left[(\beta+\mu)^{2}+r_{1} r_{3}-\left(r_{1}+r_{3}\right)(\beta+\mu)\right] } \\
& \frac{N_{0} E\left(t_{0}\right)}{\alpha I\left(t_{0}-\tau\right)} .
\end{aligned}
$$

If the condition (17) holds, then $\dot{R}\left(t_{0}\right) \geq 0$ and there does not exist a point $t_{1}>t_{0}$ such that $R\left(t_{1}\right)<0$. Hence, $R(t) \geq 0, \forall t \geq \tau$.

It follows from (13) that

$$
\begin{aligned}
u(t) \geq & 1+\frac{\beta}{\mu N_{0}} S\left(t_{0}\right) E\left(t_{0}-\tau\right)-\frac{S\left(t_{0}\right)}{\mu N_{0}}\left[k_{3}+\alpha+\mu\right. \\
& \left.+\left(\beta+\mu+\mu^{I}+\gamma\right)+\left(\mu^{I}+\gamma\right)\right] \\
& +\left[\left(\beta+\mu+k_{3}\right)\left(\beta+\mu+\mu^{I}+\gamma\right)\right.
\end{aligned}
$$

$$
\begin{aligned}
S(t) & =\frac{N_{0} c_{1}\left(\mu^{I}+\gamma-r_{1}\right)\left(\beta+\mu-r_{1}\right)\left[e^{-r_{1}(t-\tau)}-e^{-r_{3}(t-\tau)}\right]+\alpha \beta I(0) S(\tau)}{\alpha \beta I(t-\tau)} \\
& \geq 0, \forall t \geq \tau .
\end{aligned}
$$




$$
\begin{aligned}
& \left.+\left(\mu^{I}+\gamma\right)^{2}-k_{2}\right] \frac{E\left(t_{0}\right)}{\mu \alpha I\left(t_{0}-\tau\right)} \\
& +\left[k_{2}\left(\mu^{I}+\gamma\right)-k_{1}-\left(\mu^{I}+\gamma\right)^{3}\right. \\
& \left.-k_{3}\left(\mu^{I}+\gamma\right)^{2}\right] \frac{I\left(t_{0}\right)}{\mu \alpha \beta I\left(t_{0}-\tau\right)} \\
& +\frac{\delta R(t)}{\mu N_{0}} .
\end{aligned}
$$

When $k_{1}=-r_{1} r_{2} r_{3}<0, k_{2}=-\left(r_{1} r_{2}+r_{1} r_{3}+\right.$ $\left.r_{2} r_{3}\right)<0, k_{3}=-\left(r_{1}+r_{2}+r_{3}\right)<0$ and $r_{2}=\mu^{I}+\gamma$, one can get

$$
\left[\begin{array}{l}
z_{1}(t) \\
z_{2}(t) \\
z_{3}(t)
\end{array}\right]=\left[\begin{array}{c}
I(t) \\
\beta E(t)-\left(\mu^{I}+\gamma\right) I(t) \\
\beta\left[\frac{\alpha S(t) I(t-\tau)}{N_{0}}-(\beta+\mu) E(t)\right]-\left(\gamma+\mu^{I}\right)\left(\beta E(t)-\left(\gamma+\mu^{I}\right) I(t)\right)
\end{array}\right] .
$$

When the control law (15) is applied, $I(t-\tau)>\varepsilon$. Thus,

$$
\left[\begin{array}{c}
I(t) \\
E(t) \\
S(t)
\end{array}\right]=\left[\begin{array}{c}
z_{1}(t) \\
\frac{z_{2}(t)+\left(\mu^{I}+\gamma\right) z_{1}(t)}{\beta} \\
\frac{N_{0}}{\alpha \beta I(t-\tau)}\left[z_{3}(t)+\beta(\beta+\mu) E(t)+\beta\left(\gamma+\mu^{I}\right)\left[\beta E(t)-\left(\gamma+\mu^{I}\right)\right] z_{1}(t)\right]
\end{array}\right] .
$$

$$
\begin{aligned}
u(t) \geq & 1+\frac{\beta}{\mu N_{0}} S\left(t_{0}\right) E\left(t_{0}-\tau\right) \\
& +\frac{1}{\mu N_{0}}\left[r_{1}+r_{3}-\alpha-\mu\right. \\
& \left.-\left(\beta+\mu+\mu^{I}+\gamma\right)\right] S\left(t_{0}\right) \\
& +\left[(\beta+\mu)^{2}+r_{1} r_{3}-\left(r_{1}+r_{3}\right)(\beta+\mu)\right] \\
& \frac{E\left(t_{0}\right)}{\mu \alpha I\left(t_{0}-\tau\right)} \\
+ & \frac{\delta R(t)}{\mu N_{0}} .
\end{aligned}
$$

If the conditions (1) and (2) of Theorem 4 hold, then $I\left(t_{f}\right)=z_{1}\left(t_{f}\right) \leq \varepsilon<1$. If we choose $\varepsilon<$ $\min \left\{\frac{\beta}{1+\mu^{I}+\gamma}, 1\right\}$, then $E\left(t_{f}\right)<1$ which is obtained from the second equation of (23). Hence, disease is asymptotically eradicated from the host population (eventually becoming symptomatic infections) after a finite time $t_{f}$.

If the condition (17) holds, then $u(t) \geq 1$. 


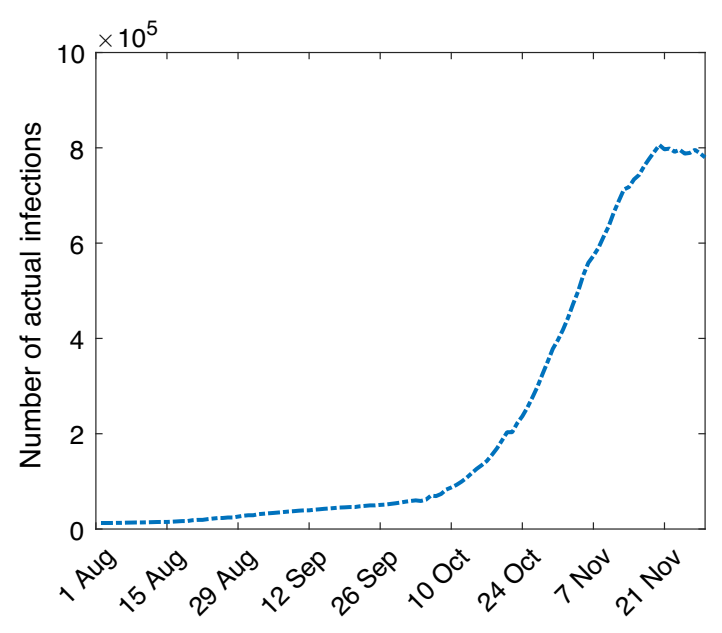

Fig. 2 The actual number of active infections in Italy

Remark 4 Since we suppose that there exist many asymptomatic infections, and we see the asymptomatic infections as vectors, the control law (15) just makes symptomatic infections and the host population (eventually becoming symptomatic infections) disappear after a finite time $t_{f}$. Hence, when there are some effective vaccines, we do not need to consider a large number of asymptomatic infections. As long as the population is vaccinated according to strategy, the virus will not cause a large number of deaths.

\section{Simulating the vaccination control of COVID-19}

This section will provide a simulation of the second outbreak of COVID-19 in Italy, and shows that the symptomatic infections can be eradicated by using the obtained vaccination control. According to reports [39], the incubation period of COVID-19 was 214 days. We take 5.8 days as time units which is the mean incubation period of COVID-19 [40]. The rate of exposed class who become infective $\beta$ is equal to $1 / 5.2=0.1923$. Suppose that the time delay $\tau=3$.As pointed out in [41], the natural death rate in Italy is equal to $12.04 \%$. The case fatality rate of COVID-19 $\mu^{I}=7.7 \%$ [42]. There are lack of real data about the proportion of recovery population who become susceptible again, transmission rate and treatment rate. Hence, we assume that $\delta=1 \%, \alpha=0.5$, and $\gamma=0.151$.

We set August 1, 2020, as time zero. Around August 1 , the second outbreak of COVID-19 occurred in Italy, and a large number of asymptomatic infections appeared. There are about 60.39 million permanent residents in Italy. The number of susceptible people is approximately equal to that of its permanent residents. Since the epidemic in Lombardy is very serious, we assume that the number of exposed people is equal to the permanent residents of Lombardy. The initial value of the infective and the recovered can obtain from published data [43]. Hence, $S(\omega)=60.09$ million, $E(\omega)=33000, I(\omega)=12646, R(\omega)=200229$, $-\tau \leq \omega \leq 0$. Figure 2 depicts the actual number of active infections in Italy according to the data of coronavirus resource center [43].

Based on the above data, it is easy to obtain that $R_{0}=2.0642>1$. As pointed out in [44], the obtained basic reproduction number $R_{0}$ is reasonable for COVID-19 in Italy. Figure 3a depicts the time evolution of system (1) when there does not exist control. Figure $3 \mathrm{~b}$ depicts the number of active infections with no control. Comparing with the actual number of active infections in Italy (see Fig. 2), the number of active infections in Italy is much higher than the actual data. The reason for this phenomenon is that we assume the medical conditions are very poor and no other methods of epidemic control have been implemented, such as wearing a mask, not going outside and keeping social distance.

If there is an effective vaccine, then the government can apply vaccination strategy (15). If we choose $\varepsilon=0.9, r_{1}=\beta+0.99 \mu, r_{2}=\mu^{I}+\gamma$ and $r_{3}=3.86\left(\mu^{I}+\gamma\right)$, then the conditions of Theorem 4 hold, and the nonnegativity of system (1) is guaranteed. The vaccination strategy (15) is applied when $t=3$ days. By direct calculation, $t_{f} \approx 66$ days. Figure $4 \mathrm{a}$ depicts the time evolution of system (1) with vaccination control (15). Figure $4 \mathrm{~b}$ depicts the number of active infections with control $u_{e}(t)$. One can see that the active infections are eradicated from the population. Moreover, the removed-by-immunity population asymptotically tracks the whole population as time tends to infinity. Figure 5 depicts the time evolution of vaccination control (15).

When the vaccination strategy (15) is applied, the proportion of the cured population to the total population will soon decline. Figure 6a illustrates the percentage of the total number of people who have recovered through treatment under different situations. As pointed out in [44], if there is no effective control strategy and the disease has spread to 350 days, then percentage of the cured population to the total population 
Fig. 3 a The time evolution of system (1) with no control when $R_{0}=2.0642$; b the number of active infections with no control when $R_{0}=2.0642$

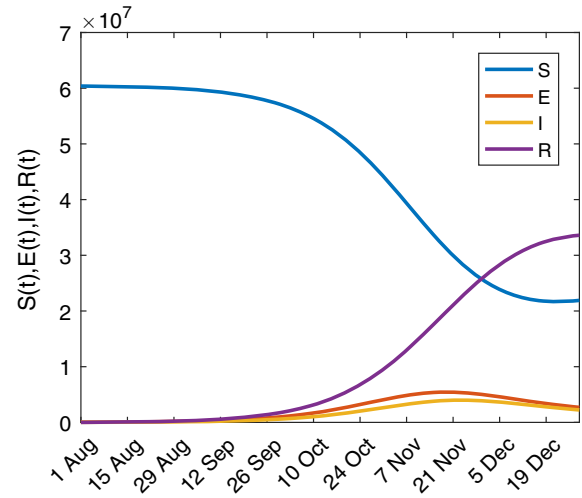

(a)

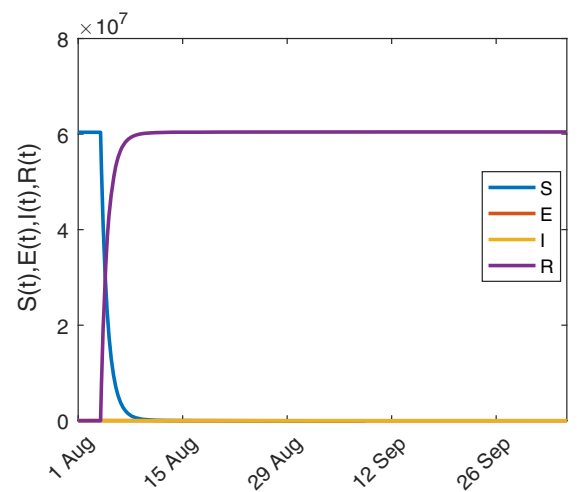

(a)

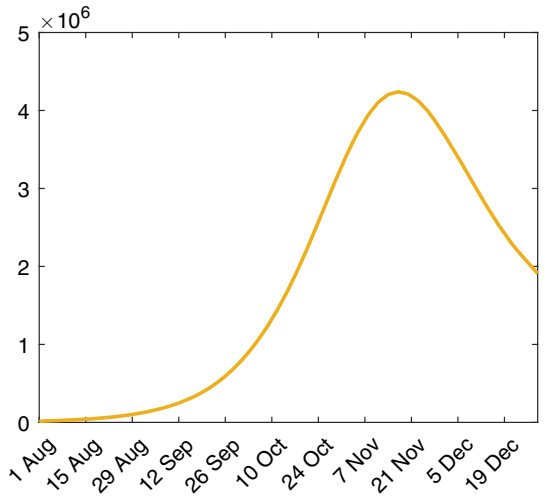

(b)

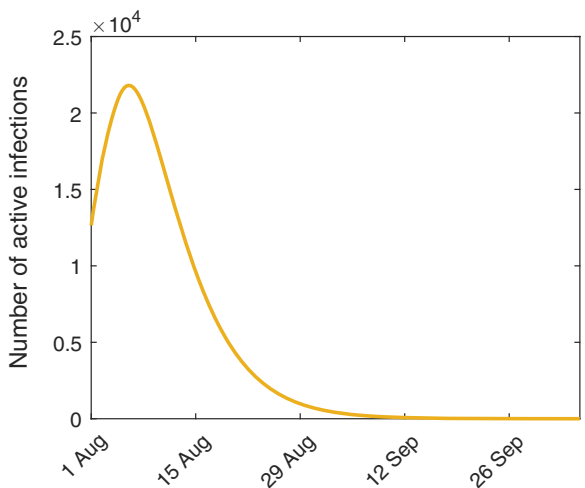

(b)

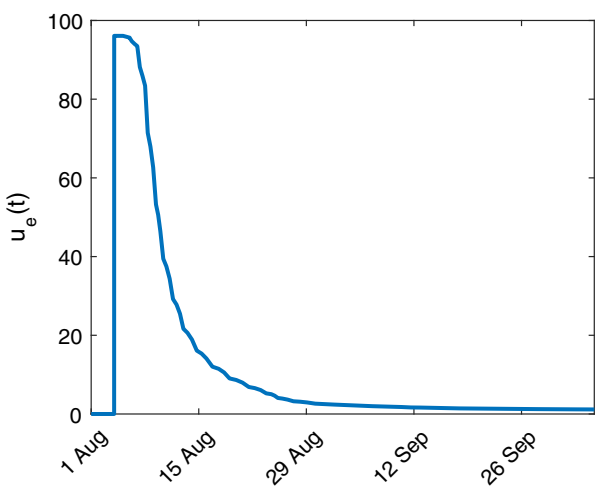

Fig. 5 Time evolution of the vaccination function $u_{e}(t)$

will approximate $0.6 \%$ (red line in Fig. 6a). The blue line is obtained from system (1) with no vaccination control. The green line is obtained from system (1) when the vaccination strategy (15) is applied. Because vaccination has been implemented, the number of people cured by treatment will become very small (Fig. 6b). Most susceptible gain immunity directly through vaccination and do not need treatment. This shows that vaccination can greatly reduce the burden on medical facilities.

In addition, if we set $\varepsilon=0.9, r_{1}=\beta-\mu$, $r_{2}=\mu^{I}+\gamma$ and $r_{3}=3\left(\mu^{I}+\gamma\right)$, then the conditions of Theorem 4 do not hold. However, one can see from Fig. 7 that the nonnegativity of system (1) is guaranteed when one applies vaccination strategy (15). This is because the condition of Theorem 4 is a sufficient condition. The vaccination strategy (15) is applied when $t=3$ days. By direct calculation, $t_{f} \approx 71$ days. Figure $7 \mathrm{a}$ depicts the time evolution of system (1) with vaccination control (15). Figure $7 \mathrm{~b}$ depicts the number of active infections with control $u_{e}(t)$. Figure 8 depicts the time evolution of vaccination control (15). 
Fig. 6 a The percentage of the total number of people who have recovered through treatment under different situations and $\mathbf{b}$ the percentage under vaccination situation

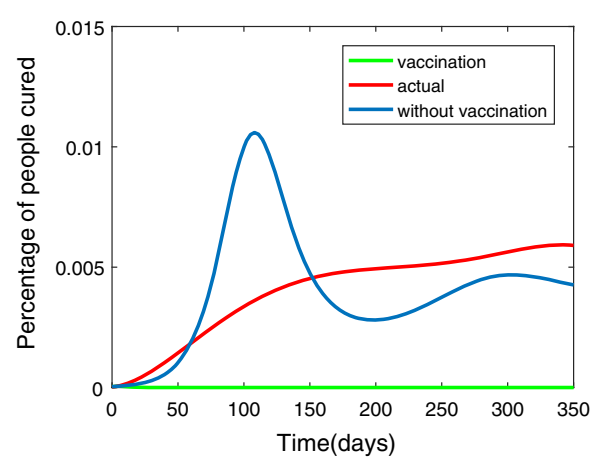

(a)

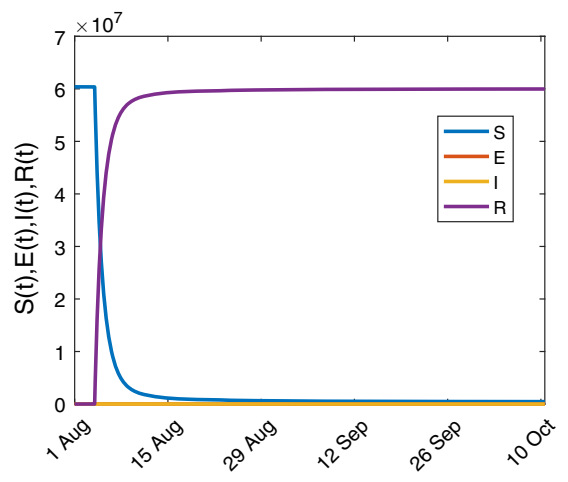

(a)

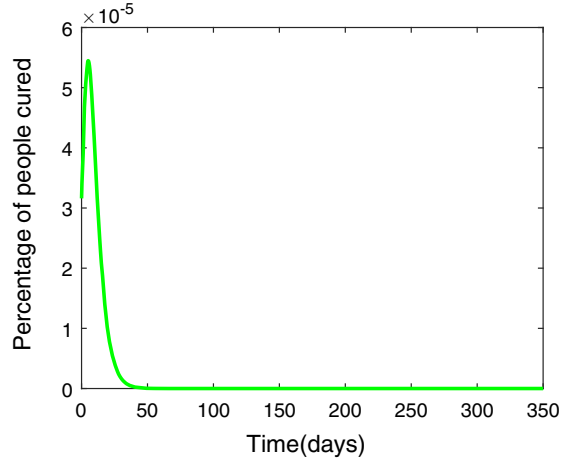

(b)

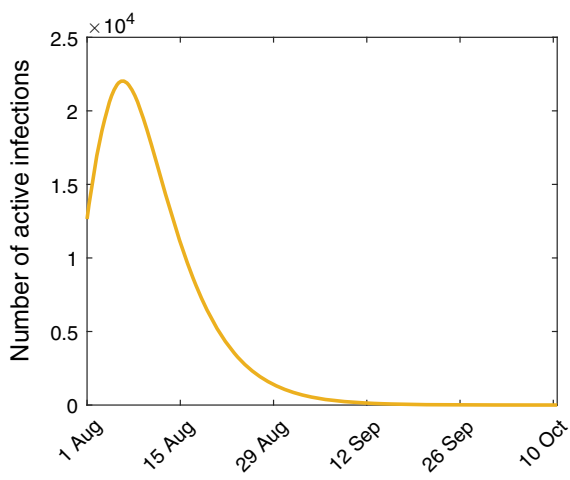

(b)

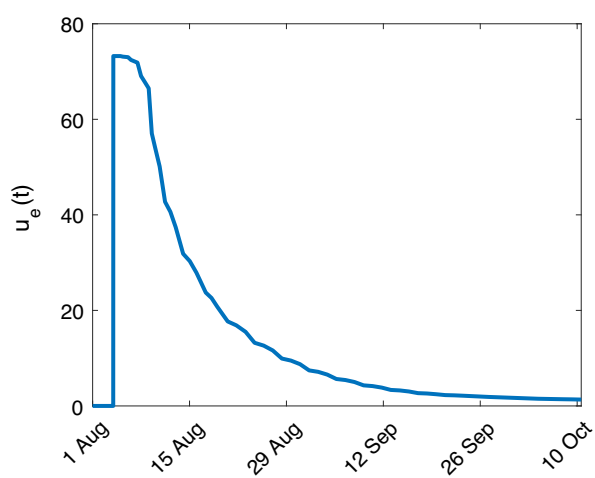

Fig. 8 Time evolution of the vaccination function $u_{e}(t)$

\section{Concluding remarks}

This paper introduced an SEIR-type epidemic model with time delay and vaccination control which assumed that the disease is high infectiousness and there exists a large number of asymptomatic infections. We have shown that the disease will persist in population when there does not exist vaccination control and the basic reproduction number $R_{0}>1$. The vaccination control was presented as a switching control based on feedback linearization technique. The proposed vaccination control can make the active infections disappear in finite time. Since COVID-19 satisfies the properties of the proposed model (high infectiousness and a large number of asymptomatic infections), we apply the proposed control to simulate the vaccination of COVID-19, and illustrate the effectiveness of the obtained results.

Acknowledgements This work was supported in part by the Natural Science Foundation of Chongqing of China under Grant cstc2019jcyj-msxmX0109, the Scientific and Technological Research Program of Chongqing Municipal Education Commission under Grant KJQN202000608, the Science and Technology Innovation Project of "Construction of Chengdu-Chongqing Double City Economic Circle" under Grant KJCX2020029.

\section{Declaration}

Conflict of interest The authors declare that they have no conflict of interest. 


\section{References}

1. Chan, J.F.W., Yuan, S., Kok, K.H., To, K.K.W., Chu, H., Yang, J., Xing, F., Liu, J., Yip, C.C.Y., Poon, R.W.S., Tsoi, H.W., Lo, S.K.F., Chan, K.H., Poon, V.K.M., Chan, W.M., Ip, J.D., Cai, J.P., Cheng, V.C.C., Chen, H., Hui, C.K.M., Yuen, K.Y.: A familial cluster of pneumonia associated with the 2019 novel coronavirus indicating person-toperson transmission: a study of a family cluster. The Lancet 395(10223), 514-523 (2020)

2. Badr, H.S., Du, H., Marshall, M., Dong, E., Squire, M.M., Gardner, L.M.: Association between mobility patterns and COVID-19 transmission in the USA: a mathematical modelling study. Lancet. Infect. Dis 20(11), 1247-1254 (2020)

3. Sjodin, H., Wilder-Smith, A., Osman, S., Farooq, Z., Rocklov, J.: Only strict quarantine measures can curb the coronavirus disease (COVID-19) outbreak in Italy, 2020. Eurosurveillance 25(13), 7-12 (2020)

4. Zhai, S., Gao, H., Luo, G., Tao, J.: Control of a multigroup COVID-19 model with immunity: treatment and test elimination. Nonlinear Dyn. (2020). https://doi.org/10. 1007/s11071-020-05961-4

5. Zheng, X., Luo, S., Sun, Y., Han, M., Liu, J., Sun, L., Zhang, L., Ling, P., Ding, Y., Jin, T., Liu, Z., Weng, J.: Asymptomatic patients and asymptomatic phases of coronavirus disease 2019 (COVID-19): a population-based surveillance study. Natl. Sci. Rev. 7(10), 1527-1539 (2020)

6. Gao, M., Yang, L., Chen, X., Deng, Y., Yang, S., Xu, H., Chen, Z., Gao, X.: A study on infectivity of asymptomatic SARS-CoV-2 carriers. Respir. Med. 169, 106026 (2020)

7. Arons, M.M., Hatfield, K.M., Reddy, S.C., Kimball, A., James, A., Jacobs, J.R., Taylor, J., Spicer, K., Bardossy, A.C., Oakley, L.P., Tanwar, S., Dyal, J.W., Harney, J., Chisty, Z., Bell, J.M., Methner, M., Paul, P., Carlson, C.M., McLaughlin, H.P., Thornburg, N., Tong, S., Tamin, A., Tao, Y., Uehara, A., Harcourt, J., Clark, S., Brostrom-Smith, C., Page, L.C., Kay, M., Lewis, J., Montgomery, P., Stone, N.D., Clark, T.A., Honein, M.A., Duchin, J.S., Jernigan, J.A., County, P.H.S.K., Team, C.C.I.: Presymptomatic SARS$\mathrm{CoV}-2$ infections and transmission in a skilled nursing facility. N. Engl. J. Med. 382(22), 2081-2090 (2020)

8. Qiu, J.: Covert coronavirus infections could be seeding new outbreaks. Nature (2020). https://doi.org/10.1038/ d41586-020-00822-x

9. Zhu, F.C., Guan, X.H., Li, Y.H., Huang, J.Y., Jiang, T., Hou, L.H., Li, J.X., Yang, B.F., Wang, L., Wang, W.J., Wu, S.P., Wang, Z., Wu, X.H., Xu, J.J., Zhang, Z., Jia, S.Y., Wang, B.S., Hu, Y., Liu, J.J., Zhang, J., Qian, X.A., Li, Q., Pan, H.X., Jiang, H.D., Deng, P., Gou, J.B., Wang, X.W., Wang, X.H., Chen, W.: Immunogenicity and safety of a recombinant adenovirus type-5-vectored COVID-19 vaccine in healthy adults aged 18 years or older: a randomised, double-blind, placebo-controlled, phase 2 trial. The Lancet 396(10249), 479-488 (2020)

10. Cook, T.M., Farrar, J.J.: COVID-19 vaccines: one step towards the beginning of the end of the global impact of the pandemic. Anaesthesia 76(4), 435-443 (2021)

11. Tanne, J.H.: COVID-19: Pfizer-BioNTech vaccine is rolled out in US. BMJ 371, 4836 (2020)
12. Cooke, K.: Stability analysis for a vector disease model. Rocky Mount. J. Math. 9(1), 31-42 (1979)

13. Al-Darabsah, I.: Threshold dynamics of a time-delayed epidemic model for continuous imperfect-vaccine with a generalized nonmonotone incidence rate. Nonlinear Dyn. 101, 1281-1300 (2020)

14. Zhao, X.Q.: Basic reproduction ratios for periodic compartmental models with time delay. J. Dyn. Diff. Equ. 29, 67-82 (2017)

15. Kumar, A., Goel, K.: Nilam: A deterministic time-delayed SIR epidemic model: mathematical modeling and analysis. Theory Biosci. 139(1), 67-76 (2020)

16. Agaba, G., Kyrychko, Y., Blyuss, K.: Time-delayed SIS epidemic model with population awareness. Ecol. Complex. 31, 50-56 (2017)

17. Liu, J., Bai, Z., Zhang, T.: A periodic two-patch SIS model with time delay and transport-related infection. J. Theor. Biol. 437, 36-44 (2018)

18. Zhu, L., Guan, G., Li, Y.: Nonlinear dynamical analysis and control strategies of a network-based SIS epidemic model with time delay. Appl. Math. Model. 70, 512-531 (2019)

19. Gao, S., Teng, Z., Xie, D.: The effects of pulse vaccination on SEIR model with two time delays. Appl. Math. Comput. 201(1), 282-292 (2008)

20. De la Sen, M., Alonso-Quesada, S., Ibeas, A.: On the stability of an SEIR epidemic model with distributed time-delay and a general class of feedback vaccination rules. Appl. Math. Comput. 270, 953-976 (2015)

21. Gao, S., Chen, L., Teng, Z.: Pulse vaccination of an SEIR epidemic model with time delay. Nonlinear Anal. Real World Appl. 9(2), 599-607 (2008)

22. Wang, W.: Global behavior of an SEIRS epidemic model with time delays. Appl. Math. Lett. 15(4), 423-428 (2002)

23. Yuan, Y., Belair, J.: Threshold dynamics in an SEIRS model with latency and temporary immunity. J. Math. Biol. 69, 875-904 (2014)

24. Yang, P., Wang, Y.: Dynamics for an SEIRS epidemic model with time delay on a scale-free network. Phys. A 527, 121290 (2019)

25. Delacruz, W.P., Savona, M.R., Thornton, J.A., Danaher, P.J.: Evidence of vaccinia dissemination despite lack of major reaction following smallpox vaccination. Vaccine 38(7), 1589-1592 (2020)

26. Agaba, G., Kyrychko, Y., Blyuss, K.: Dynamics of vaccination in a time-delayed epidemic model with awareness. Math. Biosci. 294, 92-99 (2017)

27. Farrington, C.: On vaccine efficacy and reproduction numbers. Math. Biosci. 185(1), 89-109 (2003)

28. Lv, W., Ke, Q., Li, K.: Dynamical analysis and control strategies of an SIVS epidemic model with imperfect vaccination on scale-free networks. Nonlinear Dyn. 99, 1507-1523 (2020)

29. Zhao, Z., Pang, L., Chen, Y.: Nonsynchronous bifurcation of SIRS epidemic model with birth pulse and pulse vaccination. Nonlinear Dyn. 79, 2371-2383 (2015)

30. Alonso-Quesada, S., Sen, M.D.L., Agarwal, R.P., Ibeas, A.: An observer-based vaccination control law for an SEIR epidemic model based on feedback linearization techniques for nonlinear systems. Adv. Differ. Equ. 2012(1), 1-32 (2012) 
31. De la Sen, M., Ibeas, A., Alonso-Quesada, S.: On vaccination controls for the SEIR epidemic model. Commun. Nonlinear Sci. Numer. Simul. 17(6), 2637-2658 (2012)

32. Sisodiya, O.S., Misra, O., Dhar, J.: Dynamics of cholera epidemics with impulsive vaccination and disinfection. Math. Biosci. 298, 46-57 (2018)

33. Jiao, J., Cai, S., Li, L.: Impulsive vaccination and dispersal on dynamics of an SIR epidemic model with restricting infected individuals boarding transports. Phys. A 449, 145159 (2016)

34. Zhao, Z., Chen, L., Song, X.: Impulsive vaccination of SEIR epidemic model with time delay and nonlinear incidence rate. Math. Comput. Simul. 79(3), 500-510 (2008)

35. Liu, X., Takeuchi, Y., Iwami, S.: SVIR epidemic models with vaccination strategies. J. Theor. Biol. 253(1), 1-11 (2008)

36. Jiao, H., Shen, Q.: Dynamics analysis and vaccination-based sliding mode control of a more generalized SEIR epidemic model. IEEE Access 8, 174507-174515 (2020)

37. Hale, J., Lunel, S.: Introduction to Functional Differential Equations, vol. 99. Springer, New York (1993)

38. Hirsch, W.M., Hanisch, H., Gabriel, J.P.: Differential equation models of some parasitic infections: methods for the study of asymptotic behavior. Commun. Pure Appl. Math. 38(6), 733-753 (1985)

39. Yang, Z., Zeng, Z., Wang, K., Wong, S.S., Liang, W., Zanin, M., Liu, P., Cao, X., Gao, Z., Mai, Z., Liang, J., Liu, X., Li, S., Li, Y., Ye, F., Guan, W., Yang, Y., Li, F., Luo, S., Xie, Y., Liu, B., Wang, Z., Zhang, S., Wang, Y., Zhong, N., He, J.: Modified SEIR and AI prediction of the epidemics trend of COVID-19 in China under public health interventions. J. Thorac. Dis. 12(3) (2020)
40. Li, Q., Guan, X., Wu, P., Wang, X., Zhou, L., Tong, Y., Ren, R., Leung, K.S., Lau, E.H., Wong, J.Y., Xing, X., Xiang, N., Wu, Y., Li, C., Chen, Q., Li, D., Liu, T., Zhao, J., Liu, M., Tu, W., Chen, C., Jin, L., Yang, R., Wang, Q., Zhou, S., Wang, R., Liu, H., Luo, Y., Liu, Y., Shao, G., Li, H., Tao, Z., Yang, Y., Deng, Z., Liu, B., Ma, Z., Zhang, Y., Shi, G., Lam, T.T., Wu, J.T., Gao, G.F., Cowling, B.J., Yang, B., Leung, G.M., Feng, Z.: Early transmission dynamics in Wuhan, China, of novel coronavirus-infected pneumonia. N. Engl. J. Med. 382(13), 1199-1207 (2020)

41. Carcione, J.M., Santos, J.E., Bagaini, C., Ba, J.: A simulation of a COVID-19 epidemic based on a deterministic SEIR model. Front. Public Health 8, 230 (2020)

42. Lazzerini, M., Putoto, G.: COVID-19 in Italy: momentous decisions and many uncertainties. The Lancet Global Health 8(5), e641-e642 (2020)

43. CENTER, C.R.: COVID-19 dashboard by the center for systems science and engineering (CSSE) at Johns Hopkins University (JHU). https://coronavirus.jhu.edu/map.html (2020)

44. Giordano, G., Blanchini, F., Bruno, R., Colaneri, P., Colaneri, M.: Modelling the COVID-19 epidemic and implementation of population-wide interventions in Italy. Nat. Med. 26, 855-860 (2020)

Publisher's Note Springer Nature remains neutral with regard to jurisdictional claims in published maps and institutional affiliations. 